\title{
Actividades transversales entre materias de primer curso de grado y trabajo en equipo para aumentar la motivación del alumno Cross-cutting activities between first-year undergraduate subjects and teamwork to increase the student motivation
}

\author{
David Fonseca $^{1}$, Silvia Necchi ${ }^{1}$, Marian Alaez ${ }^{2}$, Susana Romero ${ }^{3}$, Efraim Centeno ${ }^{3}$ \\ \{David.fonseca, Silvia.necchi\}@salle.url.edu, \{Marian.alaez, Sromeroyesa\}@deusto.es, Efraim.centeno@itt.comillas.edu \\ ${ }^{1}$ Departamento de Arquitectura \\ (GRETEL) \\ La Salle, Universitat Ramon Llull \\ Barcelona, España \\ ${ }^{4}$ Instituto de Investigación \\ Tecnológica \\ Universidad Pontificia de Comillas \\ Madrid, España
}

\begin{abstract}
Resumen- Motivar al alumno de primer curso de grado, no solo mejora su grado de satisfacción y su rendimiento, sino que puede prevenir el riesgo de abandono temprano de los estudios. En este sentido, para potenciar la motivación se antoja como estrategia importante trabajar las competencias específicas desde un abordaje transversal, menos específico y que permita al alumno vislumbrar las potencialidades de sus estudios y de sus relaciones entre iguales. El presente artículo muestra el resultado de dos actividades transversales realizadas en el primer curso del grado de Estudios en Arquitectura, donde el trabajo en equipo es esencial para su entrega. Los resultados han permitido identificar tanto fortalezas como debilidades de cada práctica gracias a su enfoque mixto (cuantitativo / cualitativo), lo que permitirá un rediseño de las actividades para el siguiente curso.
\end{abstract}

Palabras clave: competencias transversales, trabajo en equipo, innovación pedagógica, estudios mixtos.

Abstract- Motivating first-year undergraduate students not only improves their degree of satisfaction and performance, but can also prevent the risk of early dropout. In this sense, to enhance motivation, it seems more important to work on specific competencies from a transversal point of view, less specific approach that allows students to glimpse the potential of their studies and their relationships among peers. This article shows the results of two transversal activities carried out in the first year of the Degree in Studies of Architecture, where teamwork is essential for its delivery. The results have allowed to identify both strengths and weaknesses of each practice thanks to its mixed approach (quantitative / qualitative), which will allow a redesign of the activities for the next course.

Keywords: transversal competencies, teamwork, pedagogical innovation, mixed studies.

\section{INTRODUCCIÓN}

Los primeros cursos universitarios, suelen ser cursos donde se prepara al alumno de forma más genérica en los conceptos básicos de materias específicas. La heterogeneidad de los perfiles de entrada (Beqiri, Chase, \& Bishka, 2009; Rodenbusch, Hernandez, Simmons, \& Dolan, 2016), se ha visto agravada por el cierre en confinamiento del curso preuniversitario 2019-2020. Esta situación global se ha vinculado y está en proceso de estudio respecto a la motivación del estudiante. La falta de interacciones, y el seguimiento en virtual, genera un grado de desconocimiento y desmotivación peligroso para su seguimiento $\mathrm{y}$, por consiguiente, en su potencial rendimiento (García-Peñalvo, Corell, Abella-García, \& Grande, 2020; García \& Corell, 2020). En este sentido, un enfoque para mitigar el impacto inicial de los estudios de grado puede ser el diseño de las asignaturas, actividades y/o prácticas más introductorias centradas en las competencias genéricas. Un diseño más transversal en el trabajo de las competencias específicas mediante metodologías de trabajo más genéricas se correlaciona directamente con un aumento del rendimiento académico (Fonseca \& García-Peñalvo, 2019; Fonseca, Martí, Redondo, Navarro, \& Sánchez, 2014; Fonseca, Redondo, \& Villagrasa, 2015; Necchi, Peña, Fonseca, \& Arnal, 2020; Sanchez-Sepulveda et al., 2020).

La presente investigación es fruto de un proyecto de Investigación concedido por la asociación Aristos Campus Mundus (ACM, https://aristoscampusmundus.net/) con el fin de investigar cómo las aproximaciones transversales en estudiantes de grado mejoran su motivación. Esta mejora no solo va ligada a la motivación, de la cual se ha comprobado ampliamente su vinculación en la mejora curricular, sino lógicamente en el progreso de la autonomía del estudiante. Un dominio de competencias transversales a lo largo de sus estudios, no solo va a mejorar la competencia general del alumno, sino su futura capacitación profesional (Peña, Fonseca, \& Martí, 2016; Peña, Fonseca, Marti, \& Ferrándiz, 2018; Bezanilla et al., 2019).

El estudio analiza y compara dos actividades concretas realizadas en el primer curso de grado de Estudios en Arquitectura, donde los estudiantes deben realizar una serie de entregas basadas en el trabajo en equipo. Los trabajos potencian habilidades específicas de materias en un trabajo multidisciplinar con visitas y explicaciones in-situ en las zonas de actuación. Este tipo de trabajos se engloban en la actividad académica denominada Aula-BCN (Fonseca et al., 2019), la cual se integra como metodología activa en el proceso de cambio educativo propuesto por el NCA (Nuevo Contexto de Aprendizaje) (ARLEP, 2020), el cual se está implementando en 
todas las escuelas de La Salle a nivel estatal, desde primaria hasta la universidad, pasando por todas las etapas educativas.

En el siguiente apartado se muestra el contexto global y marco del proyecto, para pasar en el apartado 3 a describir las píldoras evaluadas. En la sección 4 mostramos los principales resultados obtenidos para cerrar con la discusión de la propuesta.

\section{CONTEXTO}

Numerosos estudios (Kahu \& Nelson, 2018) demuestran que los estudiantes que ingresan en la universidad hoy en día son muy diferentes de los profesores que les enseñan, tanto en lo que respecta a las competencias previas como a la actitud y la motivación para adquirir otras nuevas (Bunce, Flens, \& Neiles, 2010). Esto lleva a las universidades a considerar la necesidad de tener en cuenta las características y opiniones de los estudiantes para que puedan tener éxito en sus estudios. Entre los aspectos que mejoran la motivación de los estudiantes se encuentran las metodologías para mantener su atención, las cuales principalmente deben ser activas para mejorar sustancialmente su rendimiento (Freeman et al., 2014). Con el objetivo de mejorar su rendimiento, se encuentran trabajos (Partanen, 2020) que demuestran que dicha mejora es aún mayor con la interacción directa entre compañeros y profesores. En otras palabras, aunque al principio los alumnos se muestren reacios a trabajar en equipo y teman presentar sus ideas a sus compañeros, el desarrollo de estas competencias repercute no sólo en su motivación, sino también en la experiencia y los resultados finales.

A partir de estas premisas iniciales se diseñó el proyecto "Improving social and collaborative competences of undergraduate students using active methodologies. A mixed assessment approach", aprobado para su financiación en la VI Convocatoria de Proyectos de Investigación promovidos por la asociación de Aristos Campus Mundus, convocatoria 2020. El objetivo del proyecto se centra en desarrollar píldoras educativas basadas en el trabajo en equipo y en la comunicación de las ideas a desarrollar, sin perder de vista la motivación. De esta forma se consigue que los alumnos desde el inicio de sus estudios universitarios, sean conscientes del enorme potencial del trabajo en equipo, basado en las sinergias, así como de las claves que favorecen su eficacia. La idea subyacente es que el trabajo en equipo debe ser cuidadosamente alimentado desde el inicio de la formación del grupo hasta su proceso de comunicación y evaluación final.

En esta competencia se pueden distinguir tres niveles de dominio. El primero, que se ha considerado adecuado para los alumnos de primer curso, se refiere a la responsabilidad que deben mostrar los miembros del equipo en la realización de las tareas en el tiempo previsto y en la priorización de los objetivos del equipo sobre los intereses individuales. El segundo y tercer nivel, que se dejarían para cursos superiores, se refieren, respectivamente, a la participación e implicación en los objetivos del equipo, a la garantía de un buen clima y al liderazgo del equipo.

Para la evaluación de las píldoras se ha tomado como referencia el instrumento validado del MUSIC ${ }^{\circledR}$, el cual mide 5 indicadores de forma ponderada. Este modelo considera las diferentes variables que pueden influir en la motivación, tanto las consideradas como positivas -que producen un aumentocomo las que evitan la insatisfacción. También considera el papel que juegan los diferentes agentes, como el profesorado, y los factores intrínsecos de los propios estudiantes, como el hecho de que les gusten o no los retos y tareas que se les asignan. Este modelo consta de diferentes cuestionarios, habiéndose seleccionado el más adecuado al contexto a evaluar $\mathrm{y}$, dentro de éste, seleccionado las preguntas que se han considerado apropiadas para la experiencia a medir. El objetivo es identificar los puntos fuertes y débiles de las acciones educativas desde el punto de vista de la motivación y el compromiso de los estudiantes.

Las preguntas que se han elegido tienen en cuenta la percepción del alumno sobre la utilidad de las actividades y la importancia de los conocimientos adquiridos para su futuro, su capacidad para realizarlas con éxito y obtener una alta calificación, el atractivo de los métodos de enseñanza utilizados, el disfrute que obtendrá de las actividades, su control sobre las mismas y el papel de la actitud del profesor en su realización. Los parámetros obtenidos son cuantitativos y están ampliamente validados, aunque el modelo subraya que deben considerarse de forma independiente.

\section{DESCRIPCIÓN}

La presente propuesta se ha ido depurando desde su primera edición en el curso 2016-17, y de forma más coordinada en el curso 2017-18 (Fonseca et al., 2019). Básicamente, las prácticas a realizar se basan en actividades posteriores a visitas/rutas in situ a los lugares que contextualizan la actividad con la asistencia de profesores de diversas materias. Estas salidas, no solo buscan mejorar las sinergias entre las asignaturas y los profesores, sino incrementar el interés y mejorar los métodos de aprendizaje basados en el estudio de casos reales, acercando conceptos arquitectónicos fuera de las lecciones magistrales. Por otro lado, estas actividades, buscan de una forma soslayada que la motivación del estudiante de primer curso se refuerce, y que a pesar de que al comienzo de los estudios abunden las asignaturas básicas y más instrumentales, puedan entrever la dimensión que alcanzará su profesión, de manera que el potencial abandono temprano en primer curso disminuya.

La presente propuesta también se enmarca dentro de las líneas de actuación del NCA (Nuevo Contexto de Aprendizaje), modelo pedagógico basado en la implantación de métodos de aprendizaje activos, el cual está en implantación a nivel internacional por todo tipo de estamentos ligados a La Salle (colegios de primaria, secundaria, bachillerato, formación profesional y universidad).

El artículo se centra en mostrar el enfoque e implementación de dos de las píldoras diseñadas y analizar los resultados obtenidos en el caso de estudio de las visitas transversales de primer curso de arquitectura.

\section{A. Píldora 1: Escuelas de la Sagrada Familia. Contexto histórico y trabajo práctico.}

Las escuelas son una construcción de 1909-1910, un edificio provisional, económico y de rápida ejecución para resolver una necesidad: escolarizar a los hijos de los trabajadores del Templo. Su propio emplazamiento, dentro del solar donde se levantaba la Sagrada Familia, las condenaba a desaparecer a medida que avanzaban las obras. Pero esta condición de obra menor, no impidió que Gaudí planteara una geometría audaz, propia de la búsqueda y experimentación de su última etapa, centrada en la construcción de La Sagrada Familia. En 1939 y 
1938 las escuelas fueron quemadas y se reconstruyeron en 1940 por Francesc de Paula Quintana y Vidal, uno de los arquitectos que posteriormente ha dirigido la obra del Templo. En 1995 el crecimiento del Templo coincidió con el espacio físico de las escuelas, mutilándolas. En el año 2002, la junta constructora del Templo reubica las escuelas en su emplazamiento actual, delante de la fachada de la Pasión, rotada $90^{\circ}$ respecto al emplazamiento original, como se observa en la Fig. 1.

La práctica propuesta consiste en la realización de una maqueta sobre alguna de las superficies regladas que utiliza Gaudí en las escuelas o en la Sagrada Familia. Las superficies regladas y sus ejemplos de utilización son: Hiperboloide hiperbólico (altar mayor), parábolas (sección), paraboloide hiperbólico (claustro), conoide (techo y paredes de las escuelas), y helicoide (escaleras de las torres). El formato de entrega de la maqueta será libre, con la restricción de tamaño en soporte rígido de medida A3 $(29,7 \times 42 \mathrm{~cm})$. En este mismo soporte se puede incorporar la información gráfica y las imágenes que sirven para identificar la posición de la superficie en los edificios estudiados y los componentes del grupo.

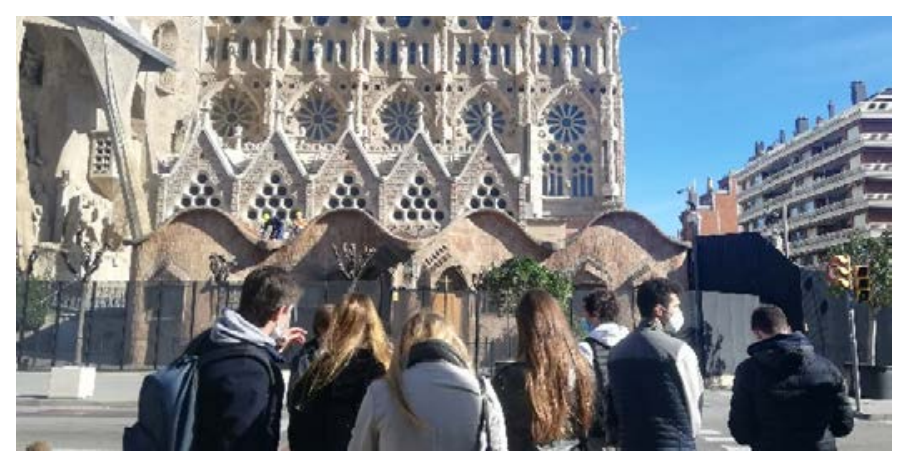

Figura 1. Clase-visita Escuelas de la Sagrada Familia (Fuente: el autor).

Las maquetas deben servir para estudiar las figuras geométricas que las originan y las rectas generatrices que forman sus superficies. El objetivo pedagógico es entenderlas desde una visión analítica y razonar cómo se pudieron ejecutar, especialmente con la tecnología del momento que fueron hechas o pensadas.

Las asignaturas que harán seguimiento de esta práctica son: Física I, Análisis Arquitectónico, Expresión Gráfica, Matemáticas y Geometría Descriptiva.

\section{B. Píldora 2: 22@, de Plaza "les Glories" al mar. Contexto histórico y trabajo práctico.}

Con el advenimiento de la democracia a los ayuntamientos, en 1979 el consistorio del alcalde Narcís Serra nombró a Oriol Bohigas Delegado de Urbanismo del ayuntamiento de Barcelona. A partir de dicho nombramiento, se produjo un periodo de fuerte inversión pública en la ciudad que conllevó un cambio de su fisonomía, y una proyección de Barcelona en el ámbito internacional. Oriol Bohigas es una figura clave por su capacidad de orientar estas inversiones, que culminarán con el hito de los Juegos Olímpicos de 1992. Entre las muchas intervenciones de este periodo, por proximidad con el ámbito donde se sitúa esta comunicación, destaca la Vila Olímpica, que se convirtió en un nuevo barrio de la ciudad frente al mar. Culminar la Vila Olímpica permitió descubrir el potencial que había a continuación, el barrio del Poble Nou.
Las ciudades han aprovechado los grandes eventos para acometer actuaciones de remodelación urbana, como el Fòrum de les Cultures en el 2004. Tomando como punto de partida el Fòrum, se ha recuperado toda la zona del Besós, se está regenerando todo el barrio del Poble Nou, ocupado por fábricas en desuso, y se ha construido el Barrio de Diagonal Mar, dotando a la ciudad de nuevos parques y espacios de ocio para los ciudadanos. Todas estas actuaciones han supuesto un cambio de la fisonomía del frente marítimo de la ciudad. La actuación que cierra esta transformación en el norte del frente marítimo es la explanada polivalente proyectada por José Antonio Martínez La Peña y Elías Torres (2000- 2004), que culmina con una gran placa fotovoltaica que conecta con la ciudad de Badalona.

El Distrito22@ tiene una superficie de unas 200 hectáreas. Se han desplegado nuevos instrumentos urbanísticos con el objetivo de transformar el antiguo suelo industrial del Poblenou para convertirlo en un área de concentración estratégica de actividades intensivas en conocimiento. A la vez, en 22@se está creando un nuevo modelo de ciudad compacta donde las empresas, universidades, centros de investigación, de formación y de transferencia tecnológica conviven con viviendas, equipamientos y zonas verdes. Para conseguir este objetivo se desarrollan proyectos destinados a promover la competitividad y la proyección internacional en sectores estratégicos: Media, Tecnologías de la Información y la Comunicación (TIC), Tecnologías Médicas (TecMed), Energía y Diseño.

\section{22@, de Plaza "les Glories” al mar. Trabajo práctico.}

Organizar un territorio es dotarlo de una estructura determinada, es intervenir sobre un terreno existente para generar un nuevo territorio. Organizar es también disponer algo para conseguir un fin determinado; disponer unas reglas para que se integren las partes entre ellas y las mismas sobre el territorio. El objetivo de la práctica es el estudio volumétrico y en sección de un entorno cercano a las instalaciones del Fórum de Barcelona. Se pide modelar, con volúmenes básicos diferenciados, todas las edificaciones del entorno y los desniveles del terreno hasta el nivel del mar, incluido todo el espigón y la Ronda Litoral. El objetivo de este análisis es identificar las ideas fuerza de este entorno no urbanizado de la ciudad para el futuro diseño de un campus de surf (último proyecto de la asignatura de Análisis Arquitectónica), según la zona de trabajo indicada en la Fig. 2.

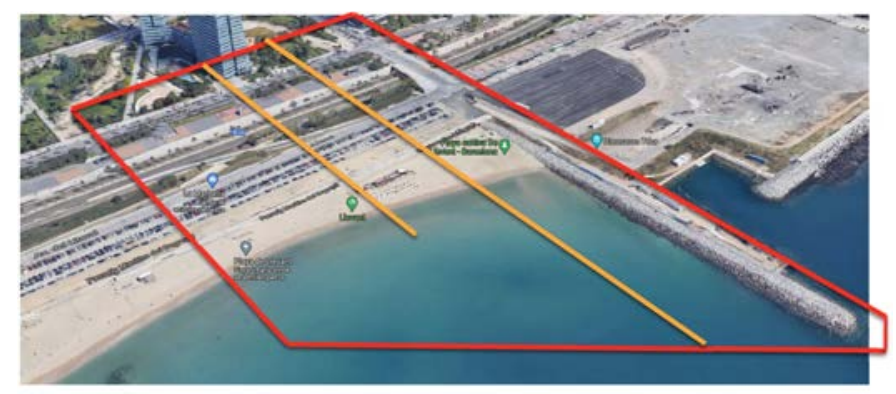

Figura 2. Zona de trabajo de la visita (Forum) (Fuente: Google Maps con edición del autor).

De forma concreta se deben generar: volúmenes sólidos e individuales para cada elemento de la zona de estudio organizados por tipo de material o uso, un volumen unido y global de todo el entorno de estudio, sección básica $\mathrm{y}$ 
automática de los dos tipos de volúmenes (individuales y agrupado), crear una sección 2D post editada con detalle, espesores, vegetación y mobiliario urbano, y un plano de situación a escala.

El formato de entrega son dos láminas DIN A3 horizontales con composición libre. El objetivo de los diversos elementos a modelar y dibujar se centra en practicar la personalización de los detalles en función de la escala para representar las conclusiones del análisis del entorno (identificación de las ideas iniciales del proyecto). Los planos deben contener: una axonometría (en perspectiva) mostrando los volúmenes diferenciados y semitransparentes que permita entender el entorno desde la torre residencial hasta al mar, una axonometría seccionada (ortogonal o en perspectiva), con la mirada hacia Badalona, donde se indique claramente la parte seccionada, y la sección $2 \mathrm{D}$ post editada con detalle, espesores, vegetación y mobiliario urbano, seccionando por la mitad del edificio de la zona ajardinada, hasta pasados unos $20 \mathrm{~m}$ de línea del mar. La escala a presentar en el DinA3 será aquella que mejor se ajuste a la presentación global (1/500 o 1/1000).

\section{Resultados}

En la Figura 3, se puede ver la exposición resultante de las entregas del ejercicio de las Escuelas de la Sagrada Familia y una selección de cinco maquetas entregadas de dicho ejercicio.

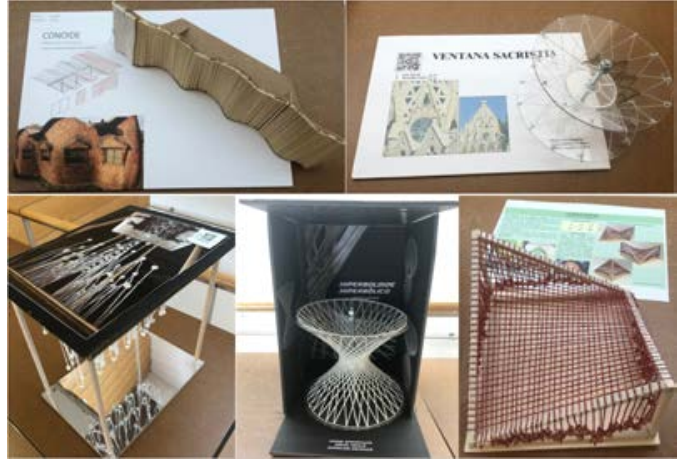

Figura 3. Cinco maquetas entregadas de diferentes tipologías de superficies. Entrega Escuelas de la Sagrada Familia.

En la Figura 4, se pueden observar diferentes ejemplos de la entrega correspondiente al ejercicio 2 de la zona22@y Forum, con secciones enriquecidas, puntos de vista 3D y modelados tridimensionales. En la Figura 5, encontramos el equivalente a una práctica completa, es decir con sus dos planos PDF en A3 y los elementos solicitados en el enunciado.

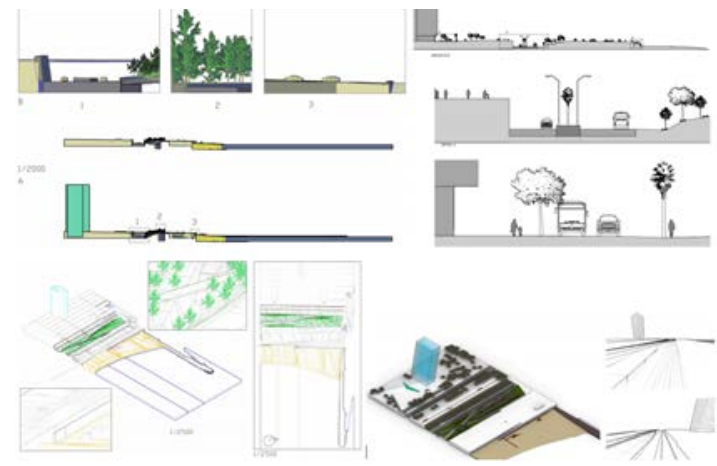

Figura 4. Ejemplo de secciones enriquecidas.

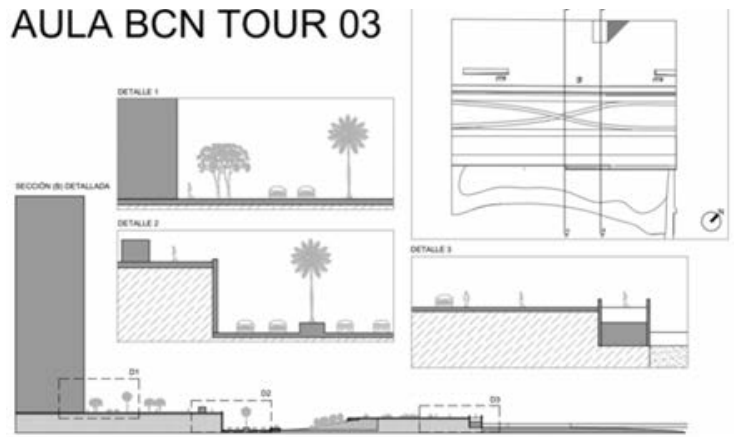

Figura 5. Ejemplo de práctica 22@-Forum completa (2 PDFs).

A continuación, se revisan los principales datos recogidos de la evaluación de las actividades y de las prácticas de forma comparada. Para la evaluación que se presenta, se han identificado dos procesos: el primero centrado en evaluar la visita (Fig. 6), la práctica (Fig. 7) y el trabajo en grupo (Fig. 8), mediante un cuestionario Likert de 5 niveles, y el segundo evaluando la motivación del estudiante mediante el instrumento del MUSIC ${ }^{\circledR}$ con una escala de 6 niveles según está definido en el instrumento, junto con una serie de preguntas de posicionamiento global (Fig. 9).

\section{CONCLUSIONES}

La muestra resultante se obtiene de las respuestas obtenidas de 26 alumnos de primer curso (sobre un total posible de 48 , lo que conforma el $54,16 \%$ ), los cuales libremente y después de ser informados rellenaron la valoración de forma anónima. Como se comprueba de las gráficas de resultados, en general se puede afirmar que la valoración de los alumnos a las dos visitas/actividades es muy positiva. Todos los indicadores relacionados con la visita (Fig. 6) se sitúan por encima del 60\% de valoraciones Adecuadas o Muy adecuadas, destacando el sexto indicador con las valoraciones más elevadas, en las que el alumno comprende las interrelaciones entre materias, justamente el objetivo de este tipo de actividades. Si se analizan las respuestas con una peor valoración (ítems 1 y 2), la información previa y los objetivos de la actividad desde un punto de vista inicial, son los aspectos identificados por los alumnos a mejorar. Centrándose en la actividad práctica (Fig. 8 ), mayoritariamente de nuevo las percepciones positivas o muy positivas se sitúan por encima del $60 \%$ de las respuestas, destacando en el aspecto positivo el formato de las entregas para cada ejercicio (cuarto ítem). En el extremo opuesto, la variable peor valorada ha sido la documentación de soporte (quinto ítem), siendo un aspecto que queda reflejado y que hay que mejorar en siguientes iteraciones. La valoración del trabajo en grupo (Fig. 8) es altamente positiva en las dos actividades, con residuos de valoraciones negativas principalmente en la primera, cuyo entregable tenía dos grandes hándicaps: la elección de la forma y del método de representación, lo cual se antoja como un aspecto de mayor necesidad de coordinación entre los alumnos. Un aspecto positivo adicional se obtiene de comparar estos resultados con la primera actividad del curso (Aláez et al., 2021), donde el porcentaje de resultados neutros y/o negativos se situaba alrededor del $30 \%$. En resumen y de los datos finales (ver Fig. 9), los alumnos se han ido motivando con este tipo de actividades y han ido mejorando en los progresos de mejora de la competencia de su trabajo en equipo, lo cual potencia el fortalecimiento de este tipo de actividades, poniendo especial atención a las primeras actividades del curso para una mejor gestión del trabajo en grupo y de los contenidos. 


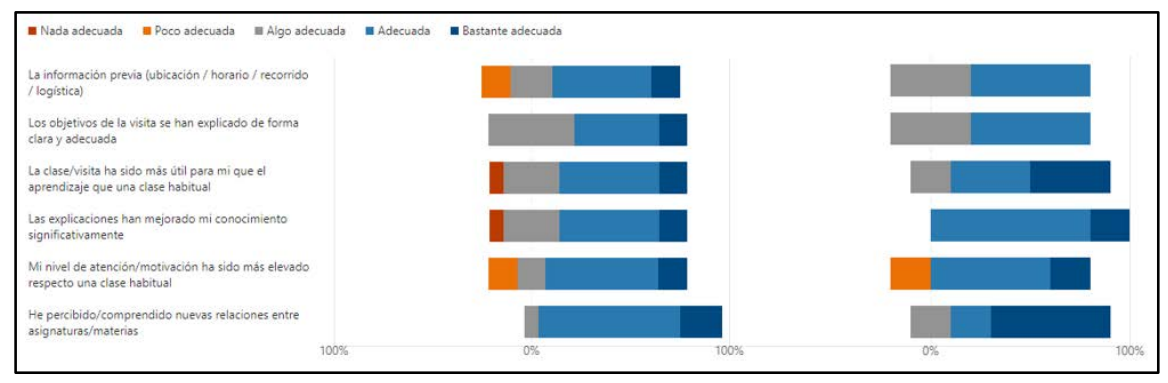

Figura 6. Valoración comparada de las dos visitas.

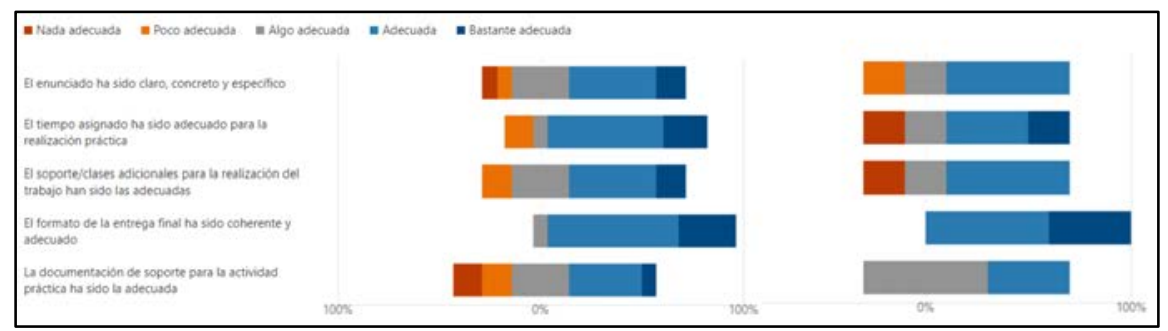

Figura 8. Valoración comparada de las dos actividades evaluativas post visita.

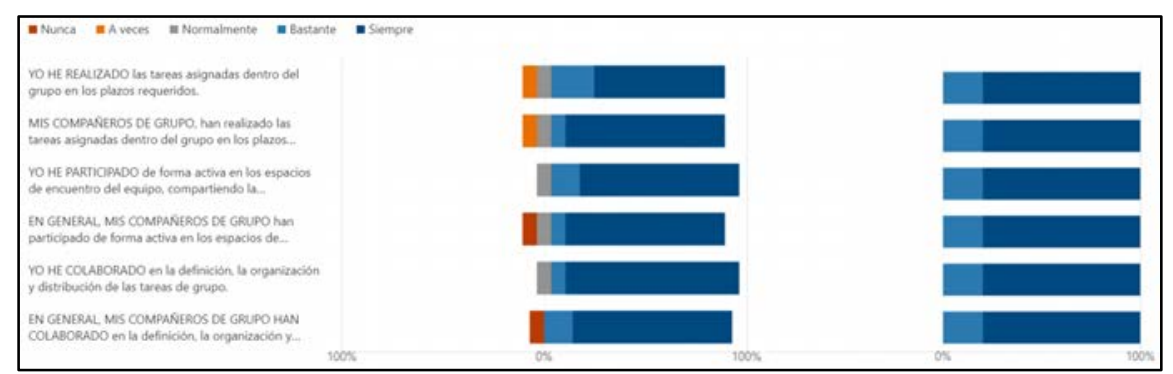

Figura 9. Valoración comparada del trabajo en grupo realizado en ambas actividades.

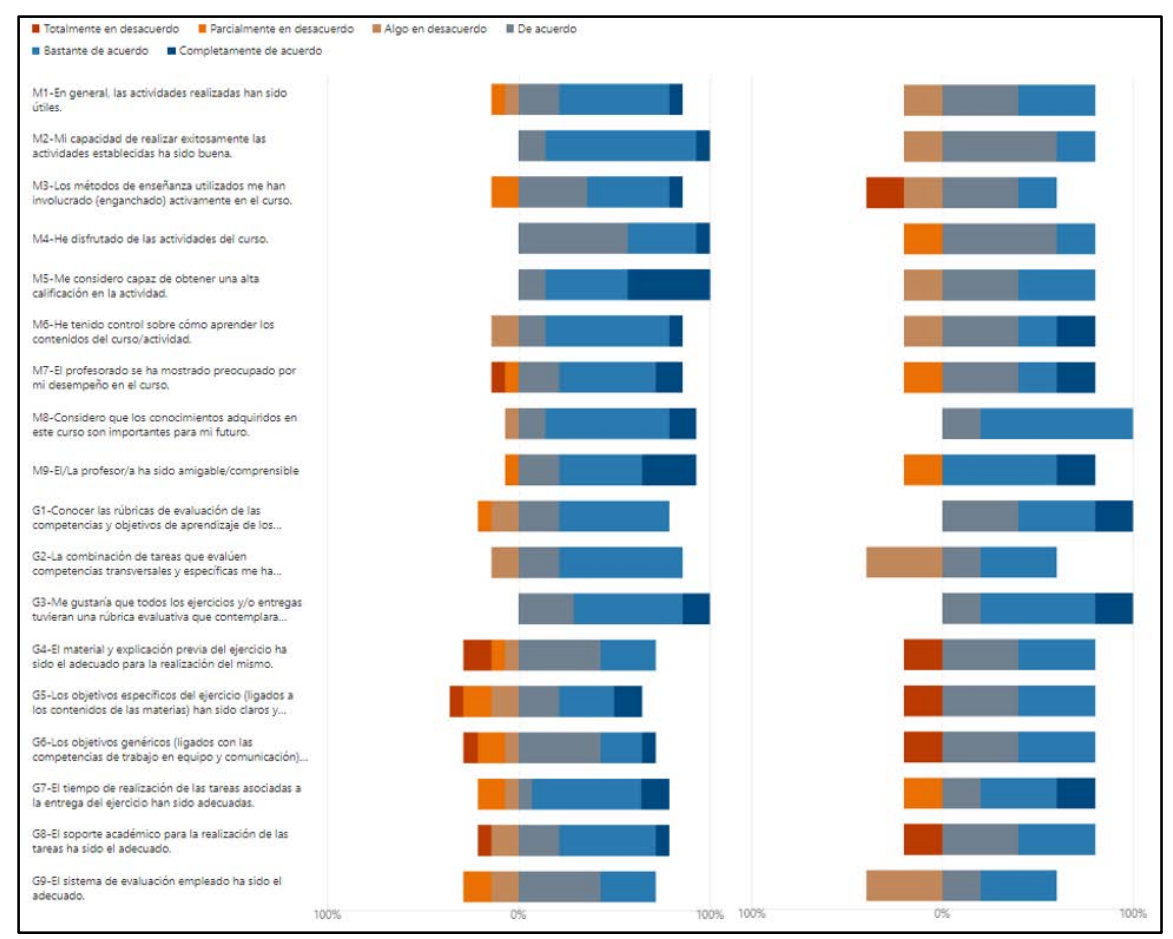

Figura 10. Evaluación global MUSIC® (preguntas M1 a M9) y de satisfacción global (G1-G9)

\section{AgRADECIMIENTOS}

Esta investigación está financiada por el proyecto "Improving social and collaborative competences of undergraduate students using active methodologies. A mixed assessment approach", concedido en la VI Convocatoria de Proyectos de Investigación ACM (Aristos Campus Mundus) 2020 con el identificador ACM2020_02. 


\section{REFERENCIAS}

Aláez, M., Romero, S., Fonseca, D., Amo, D., Peña, E., \& Necchi, S. (2021). Auto-assessment of Teamwork and Communication Competences improvement applying active methodologies. Comparing results between students of first academic year in Architecture, Economics and Engineering degrees. In I. A. Zaphiris P. (Ed.). Lecture Notes in Computer Science, vol .23 LNCS 12784, 8th Int. Conf. LCT 20201, Held as Part of the 23nd HCI Internation. https://doi.org/978-3-030-77888-0

ARLEP, L. S. D. (2020). NCA, Nuevo Contexto de Aprendizaje (La Salle A). Madrid: La Salle ARLEP.

Beqiri, M. S., Chase, N. M., \& Bishka, A. (2009). Online Course Delivery: An Empirical Investigation of Factors Affecting Student Satisfaction. Journal of Education for Business, 85(2), 95-100. https://doi.org/10.1080/08832320903258527

Bezanilla, M.J., García-Olalla, A.M., Paños, J. \& Poblete, M. (2019). A model for the evaluation of competence-based learning implementation in higher education institutions: Criteria and indicators. Tuning Journal for Higher Education, 6(2), 127-174. https://doi.org/10.18543/tjhe6(2)-2019pp127-174

Bunce, D. M., Flens, E. A., \& Neiles, K. Y. (2010). How long can students pay attention in class? A study of student attention decline using clickers. Journal of Chemical Education, 87(12), 1438-1443. https://doi.org/10.1021/ed100409p

Fonseca, D., \& García-Peñalvo, F. J. (2019). Interactive and collaborative technological ecosystems for improving academic motivation and engagement. Universal Access in the Information Society, Vol. 18, pp. 423-430. https://doi.org/10.1007/s10209-019-00669-8

Fonseca, D., Martí, N., Redondo, E., Navarro, I., \& Sánchez, A. (2014). Relationship between student profile, tool use, participation, and academic performance with the use of Augmented Reality technology for visualized architecture models. Computers in Human Behavior, $31(1)$, 434-445. https://doi.org/10.1016/j.chb.2013.03.006

Fonseca, D., Necchi, S., Simón, D., Berruezo, A., Bertocchi, J., Sánchez, M., ... de Renteria., I. (2019). Evaluación mixta de actividades transversales en el grado de Arquitectura basadas en la metodología de la "Lesson Study." In U. P. de Madrid (Ed.), Proceedings of CINAIC 2019 Congreso Internacional sobre Aprendizaje, Innovación y Cooperación (pp. 331-336). https://doi.org/10.26754/cinaic.2019.0071

Fonseca, D., Redondo, E., \& Villagrasa, S. (2015). Mixedmethods research: a new approach to evaluating the motivation and satisfaction of university students using advanced visual technologies. Universal Access in the Information Society, 14(3), 311-332. https://doi.org/10.1007/s10209-014-0361-4
Freeman, S., Eddy, S. L., McDonough, M., Smith, M. K., Okoroafor, N., Jordt, H., \& Wenderoth, M. P. (2014). Active learning increases student performance in science, engineering, and mathematics. Proceedings of the National Academy of Sciences of the United States of America, 111(23), 8410-8415. https://doi.org/10.1073/pnas.1319030111

García-Peñalvo, F. J., Corell, A., Abella-García, V., \& Grande, M. (2020). La evaluación online en la educación superior en tiempos de la COVID-19. Education in the Knowledge Society (EKS), 21(0), 26 https://doi.org/10.14201/eks.23086

García, F., \& Corell, A. (2020). La CoVId-19: ¿enzima de la transformación digital de la docencia o reflejo de una crisis metodológica y competencial en la educación superior? In Campus Virtuales (Vol. 9). Retrieved from www.revistacampusvirtuales.es

Kahu, E. R., \& Nelson, K. (2018). Student engagement in the educational interface: understanding the mechanisms of student success. Higher Education Research and Development, 37(1), 58-71. https://doi.org/10.1080/07294360.2017.1344197

Necchi, S., Peña, E., Fonseca, D., \& Arnal, M. (2020). Improving teamwork competence applied in the building and construction engineering final degree project. Int. Journal of Engineering Education, 36(1 B), 328-340.

Partanen, L. (2020). How student-centred teaching in quantum chemistry affects students' experiences of learning and motivation - A self-determination theory perspective. Chemistry Education Research and Practice, 21(1), 79 94. https://doi.org/10.1039/c9rp00036d

Peña, E., Fonseca, D., \& Martí, N. (2016). Relationship between learning indicators in the development and result of the building engineering degree final project. ACM Int. Conference Proceeding Series, 02-04-Nove, 335-340. https://doi.org/10.1145/3012430.3012537

Peña, E., Fonseca, D., Marti, N., \& Ferrándiz, J. (2018). Relationship between specific professional competences and learning activities of the building and construction engineering degree final project. International Journal of Engineering Education, 34(3), 924-939.

Rodenbusch, S. E., Hernandez, P. R., Simmons, S. L., \& Dolan, E. L. (2016). Early engagement in course-based research increases graduation rates and completion of science, engineering, and mathematics degrees. CBE Life Sciences Education, 15(2). https://doi.org/10.1187/cbe.16-030117

Sanchez-Sepulveda, M. V., Fonseca, D., García-Holgado, A., García-Peñalvo, F. J., Franquesa, J., Redondo, E., \& Moreira, F. (2020). Evaluation of an interactive educational system in urban knowledge acquisition and representation based on students' profiles. Expert Systems, 37(5). https://doi.org/10.1111/exsy.12570 